\title{
Structure probing of tmRNA in distinct stages of trans-translation
}

\author{
NATALIA IVANOVA, ${ }^{1}$ MAGNUS LINDELL, ${ }^{1}$ MICHAEL PAVLOV,${ }^{1}$ LOVISA HOLMBERG SCHIAVONE, ${ }^{2}$ \\ E. GERHART H. WAGNER, ${ }^{1}$ and MÅNS EHRENBERG ${ }^{\mathbf{1}}$ \\ ${ }^{1}$ Department of Cell and Molecular Biology, Uppsala University, S-75124 Uppsala, Sweden \\ ${ }^{2}$ Cell Biology Unit, Natural Science Section, Södertörns Högskola, S-141 89 Huddinge, Sweden
}

\begin{abstract}
Ribosomes stalled on problematic mRNAs in bacterial cells can be rescued by transfer-messenger RNA (tmRNA), its helper protein (small protein B, SmpB), and elongation factor Tu (EF-Tu) through a mechanism called trans-translation. In this work we used lead(II) footprinting to probe the interactions of tmRNA with SmpB and other components of the translation machinery at different steps of the trans-translation cycle. Ribosomes with a short nascent peptide stalled on a truncated mRNA were reacted with Ala-tmRNA.EF-Tu-GTP, SmpB, and other translation components to initiate and execute trans-translation. Free tmRNA was probed with lead(II) acetate with and without $\mathrm{SmpB}$, and ribosome bound tmRNA was probed in one of four different transtranslation states stabilized by antibiotic addition or selective exclusion of translation components. For comparison, we also analyzed lead(II) cleavage patterns of tmRNA in vivo in a wild-type as well as in an SmpB-deficient Escherichia coli strain. We observed some specific cleavages/protections in tmRNA for the individual steps of trans-translation, but the overall tmRNA conformation appeared to be similar in the stages analyzed. Our findings suggest that, in vivo, a dominant fraction of tmRNA is in complex with SmpB and that, in vitro, SmpB remains tmRNA bound at the initial steps of trans-translation.
\end{abstract}

Keywords: tmRNA; SmpB; structural probing; lead(II); trans-translation

\section{INTRODUCTION}

Transfer-messenger RNA (tmRNA, also known as SsrA and 10Sa in Escherichia coli) is an unusual RNA species with the properties of both a tRNA (Komine et al. 1994; Ushida et al. 1994) and an mRNA (Nissen et al. 1995). Together with its helper protein small protein B (SmpB) (Karzai et al. 1999; Hanawa-Suetsugu et al. 2002; Shimizu and Ueda 2002) and elongation factor Tu (EF-Tu) (Rudinger-Thirion et al. 1999), alanylated tmRNA resolves ribosome complexes stalled on defective mRNAs via a process called trans-translation (Atkins and Gesteland 1996; Keiler and Sauer 1996). Genes encoding tmRNA and SmpB are present in all known eubacterial genomes (Wower and Zwieb 1999; Keiler et al. 2000; Gaudin et al. 2002; Andersen et al. 2006), but not in either archaeal genomes or nuclear

Reprint requests to: Måns Ehrenberg, Department of Cell and Molecular Biology, Uppsala University, Box 596, 75124 Uppsala, Sweden; e-mail: ehrenberg@xray.bmc.uu.se; fax +4618530396; or Gerhart Wagner, Department of Cell and Molecular Biology, Uppsala University, Box 596, 75124 Uppsala, Sweden; e-mail: gerhart.wagner@icm.uu.se; fax +4618530396.

Article published online ahead of print. Article and publication date are at http://www.rnajournal.org/cgi/doi/10.1261/rna.451507. genomes of eukaryotes (Wower and Zwieb 1999; Keiler et al. 2000; Gaudin et al. 2002). Depending on the species, the length of bacterial tmRNA ranges between 260 and 430 nucleotides (nt). The $5^{\prime}$ and $3^{\prime}$ ends of tmRNA fold into a tRNA-like domain (TLD) that resembles the acceptor stem and the T stem-loop of tRNA ${ }^{\text {Ala }}$ (Komine et al. 1994; Ushida et al. 1994). The CCA 3' end of TLD of tmRNA is acylated with alanine by alanyl-tRNA synthetase (AlaRS) (Komine et al. 1994; Ushida et al. 1994). The TLD is connected through several RNA helices and four pseudoknots (PK1-PK4) (Felden et al. 1997) to the mRNA-like domain (MLD) of tmRNA, which contains an internal open reading frame (ORF) that encodes a short proteolysis tag (amino acid sequence: ANDENYALAA in E. coli) (Nissen et al. 1995; Keiler and Sauer 1996).

Information derived from chemical and enzymatic probing (Felden et al. 1997; Hickerson et al. 1998; Nameki et al. 1999), phylogenetic analysis (Williams and Bartel 1996; Wower and Zwieb 1999; Felden et al. 2001), and UVinduced intramolecular cross-linking (Knudsen et al. 2001) has been used to construct a secondary structure model of tmRNA (Felden et al. 1997). Lead(II) probing of tmRNA in vivo has corroborated this model and suggests similar 
tmRNA folding in vitro and in vivo (Knudsen et al. 2001; Lindell et al. 2002).

Functional interactions between tmRNA and its protein partners such as AlaRS, SmpB, EF-Tu, and ribosomal protein S1 have been studied with different techniques (Karzai et al. 1999; Rudinger-Thirion et al. 1999; Barends et al. 2000, 2001; Wower et al. 2002). It has been shown that EF-Tu•GTP forms a complex with the aminoacylated TLD of tmRNA (Rudinger-Thirion et al. 1999), and that SmpB, EF-Tu, and S1 occupy nonoverlapping binding sites on tmRNA in solution (Barends et al. 2001; Wower et al. 2002). The crystal structure of the SmpB -TLD complex from Aquifex aeolicus reveals contacts between the TLD in tmRNA and SmpB (Gutmann et al. 2003). In vitro experiments suggest that $\mathrm{SmpB}$ is essential for, and that EF-Tu greatly accelerates, the binding of tmRNA to stalled ribosomes (Barends et al. 2001; Hanawa-Suetsugu et al. 2001; Shimizu and Ueda 2002; Hallier et al. 2004). It has been proposed that S1 unwinds the secondary structure of tmRNA during its movement through the ribosome (Barends et al. 2000; Valle et al. 2003). Biochemical (Hallier et al. 2004; Ivanova et al. 2005a) and recent cryo-EM (Kaur et al. 2006) observations suggest that two copies of SmpB bind to the stalled ribosome and participate in the transtranslation process.

Trans-translation is initiated via the SmpB-dependent recognition of a stalled ribosome by the EF-Tu•GTP•AlatmRNA complex (Karzai et al. 1999; Ivanova et al. 2004). When tmRNA is arrested in a preaccommodation ribosomal complex by kirromycin, it interacts extensively with EF-Tu, two copies of SmpB, and the 30 S ribosomal subunit (Kaur et al. 2006). After EF-Tu dissociation and accommodation of the TLD of tmRNA in the ribosomal A site, the nascent peptide is transferred from the P-site tRNA to the A-site Ala-tmRNA. Subsequently, elongation factor G (EF-G) translocates the TLD of the peptidyl-tmRNA into the $\mathrm{P}$ site and the first codon of the ORF of tmRNA into the A site of the ribosome. This event results in the dissociation of both the deacylated tRNA and the truncated mRNA from the ribosome (Ivanova et al. 2005b). The A site is then filled by Ala-tRNA ${ }^{\mathrm{Ala}}$ and the nascent peptide is transferred from the peptidyl-tmRNA to the Ala-tRNA ${ }^{\mathrm{Ala}}$. In the following translocation event, peptidyl-tRNA ${ }^{\mathrm{Ala}}$ is moved into the $\mathrm{P}$ site and the TLD of tmRNA is presumably moved into the $\mathrm{E}$ site of the ribosome. At this point, the tRNA role of tmRNA is completed and the translation continues on the MLD.

While tmRNA in the preaccommodation state has been characterized in considerable detail, much less is known about its post-accommodation structure (Valle et al. 2003; Kaur et al. 2006). Furthermore, the structure and positioning of tmRNA in the states following the first translocation event in trans-translation are virtually unknown. In this study we have prepared ribosome complexes with tmRNA arrested in the pre- and post-accommodation states as well as in the states after the first and second translocation events in trans-translation. The structure of tmRNA in these complexes, in the free state and in complex with SmpB, was probed with lead(II) and DMS. We found that the interaction between tmRNA and SmpB persists during all studied steps of trans-translation and that the secondary structure of tmRNA remains largely intact during the passage of the TLD of tmRNA through the ribosome. There are, at the same time, subtle differences in the lead cleavage patterns for tmRNA in its different ribosomal states. The present observations are compared to data from recent biochemical and structural studies of tmRNA.

\section{RESULTS}

The lead(II) probe efficiently cleaves RNA in single-strand regions, loops, and bulges and may cleave flexible or breathing double-strand regions (Gornicki et al. 1989), while DMS modifies the N-1 position of adenines and the $\mathrm{N}-3$ position of cytosines in single-stranded regions of tmRNA. The lead(II) cleavages and DMS-modified nucleotides in tmRNA were identified by primer extension (see Materials and Methods). The reactivity of some nucleotides to the chemical probes was affected by the various interaction partners of tmRNA (Fig. 1; also see Fig. 5 below). These nucleotides are listed in Table 1 and are mapped to the secondary structure of tmRNA in Figure 2.

\section{Lead(II) cleavage and DMS modification of naked tmRNA}

Most of the observed lead(II) cleavages and DMS modifications in the naked tmRNA (Fig. 1; data not shown) are consistent with its secondary structure as derived from in vitro probing data (Felden et al. 1997; Hickerson et al. 1998; Nameki et al. 1999) and phylogenetic comparisons (Williams and Bartel 1996; Wower and Zwieb 1999; Felden et al. 2001). However, we observed lead(II) cleavages also in some putative double-stranded regions in h2 (nucleotides A312, A319, U320, and A34) and PK3 (U221, U222, U240, and A207), suggesting that these nucleotides were single stranded or flexible in naked tmRNA (Fig. 1; data not shown).

\section{Mapping tmRNA interaction with SmpB in vitro and in vivo}

To localize the binding site(s) of SmpB on tmRNA, lead(II) probing and DMS modification were performed either on tmRNA.SmpB complexes, formed by mixing $\mathrm{SmpB}$ and tmRNA at molar ratios from $1: 1$ to $10: 1$, or on tmRNA.SmpB complexes further purified on a Ni-NTA column (see Materials and Methods). A comparison of the lead(II) cleavage pattern of tmRNA in the presence and absence of SmpB shows a clear SmpB footprint in the TLD 


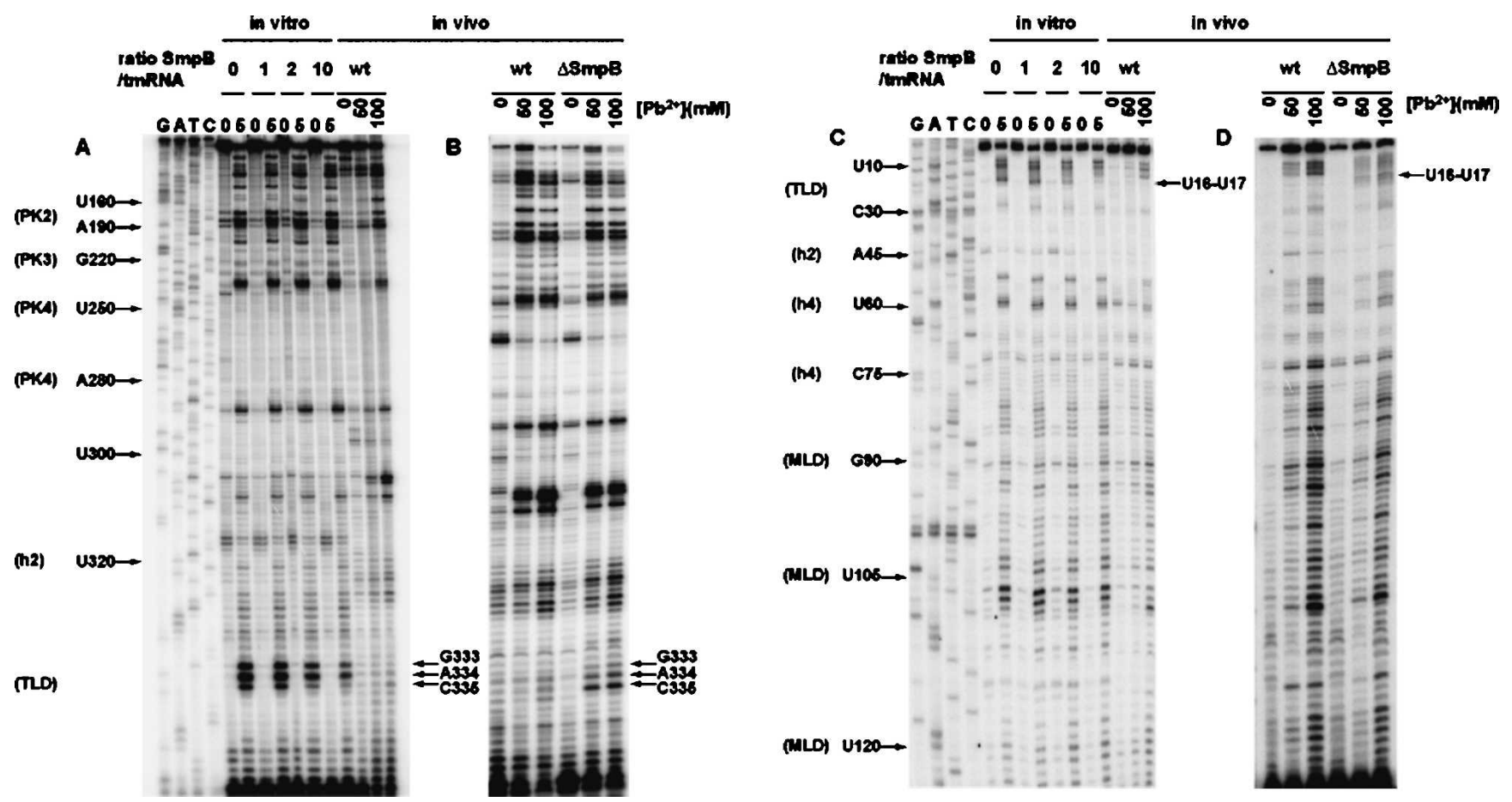

FIGURE 1. Footprinting the tmRNA interaction with $S m p B$ in vitro and in vivo. Lead(II) concentration for optimal (partial) cleavage in vitro was $5 \mathrm{mM}$ and the SmpB:tmRNA ratios were 1:1, 2:1, and 10:1. Primer extensions with tmRNA from the wild-type (wt) strain were run on the same gels for comparison $(A, C)$. In a parallel experiment, the same primer extensions after lead(II) cleavage were performed on total RNA extracted from isogenic wt and $\Delta s m p B$ strains $(B, D)$. Lead(II) concentrations used for in vivo cleavage are indicated. Two different radiolabeled primers were used for primer extension analysis: GW-TM5 $(A, B)$ and GW-TM3 $(C, D)$. Nucleotides strongly protected from lead(II) cleavage in the presence of SmpB are indicated. G, A, T, C sequencing ladders were used for identification of cleavage positions. Some of these positions are marked to simplify their identification.

of tmRNA (Fig. 1A,C). The extent of lead(II) cleavage at positions G333-C335 and U16-U17 in tmRNA decreased with increasing SmpB concentration (Fig. 1A,C), but no additional protected nucleotides were visible in tmRNA even at a 10 -fold molar excess of SmpB over tmRNA. SmpB protection against DMS modification was only seen at position A334 (data not shown). Figure 2 shows that nucleotides U16-U17 are located at the $3^{\prime}$ end of the D loop whereas nucleotides G333-C335 are part of the short connector loop near the $5^{\prime}$ end of the T stem. The SmpB "footprint" in a three-dimensional (3D) model of the $\mathrm{SmpB} \cdot \mathrm{TLD} \cdot \mathrm{EF}-\mathrm{Tu} \cdot \mathrm{GTP}$ complex is shown in Figure 3.

To map the regions of E. coli tmRNA that are affected by $S m p B$ in vivo we have analyzed cleavage patterns of tmRNA in isogenic wild-type (wt) and $\Delta s m p B$ strains after treatment with lead(II) acetate (see Materials and Methods). The nucleotides specifically protected by $\mathrm{SmpB}$ in the wt but not in the $\Delta s m p B$ strain (Fig. 1, $\Delta s m p B$ ), were identical to those protected by $\mathrm{SmpB}$ in vitro (Fig. 1B,D; G333-C335 and U16-U17). Other, less-pronounced differences between the wt and $\Delta s m p B$ strains in their reactivity to the lead(II) acetate probe can also be seen in Figure 1D. For example, nucleotides C66-U68, located within the pseudoknot structure in PK1, were weakly protected from
lead(II) cleavage in the $\Delta s m p B$ strain but not in the wt strain.

\section{Mapping the tmRNA interaction with SmpB at different stages of trans-translation}

To analyze the interaction between tmRNA and SmpB in trans-translation, we prepared four functional ribosomal complexes (RCI-RCIV) (Fig. 4) arrested at different steps of trans-translation (see Materials and Methods for details).

The "preaccommodated" RCI complex was prepared by reacting ribosomes containing the fMFTI peptide in the ribosomal P site with Ala-tmRNA, SmpB, and EF-Tu•GTP in the presence of kirromycin. The "post-accommodated" RCII was prepared in the same way as RCI but without kirromycin. In this complex the fMFTI peptide was transferred to the Ala-tmRNA bound in the A site. The posttranslocated RCIII complex was obtained from RCII by addition of EF-G to translocate the fMFTIA-tmRNA into the P site of the ribosome (Ivanova et al. 2005b). Finally, the "twice-translocated" RCIV complex, containing fMFTIAA in the P site and the deacylated TLD of tmRNA probably in the $\mathrm{E}$ site of the ribosome, was obtained from RCIII by adding Ala-tRNA ${ }^{\text {Ala }}$ to the reaction mix to decode 
TABLE 1. Lead(II)-induced cleavages of tmRNA in different ribosomal complexes

\begin{tabular}{|c|c|c|c|c|c|c|}
\hline $\begin{array}{l}\text { Region in } \\
\text { tmRNA }\end{array}$ & $\begin{array}{l}\text { Nucleotide } \\
\text { position }\end{array}$ & Naked & $\mathrm{RCl}$ & RCII & RCIII & RCIV \\
\hline TLD & $\mathrm{C} 335^{\mathrm{a}}$ & $6.2 \times^{b}$ & $1.0 \times$ & $1.0 \times$ & $2.5 \times$ & $1.8 \times$ \\
\hline TLD & A334 & $21.5 \times$ & $1.0 \times$ & $1.5 \times$ & $5.0 \times$ & $2.9 \times$ \\
\hline TLD & G333 & $23.5 \times$ & $2.1 \times$ & $1.0 \times$ & $2.4 \times$ & $2.3 \times$ \\
\hline PK4 & A298 & $1.7 \times$ & $1.8 \times$ & $1.3 \times$ & $4.7 \times$ & $1.0 \times$ \\
\hline PK4 & A294 & $1.4 \times$ & $1.5 \times$ & $1.0 \times$ & $5.4 \times$ & $1.0 \times$ \\
\hline PK3 & A197 & $3.8 \times$ & $1.0 \times$ & $1.0 \times$ & $1.0 \times$ & $1.0 \times$ \\
\hline PK2 & A185 & $9.0 \times$ & $1.5 \times$ & $3.6 \times$ & $1.0 \times$ & $1.0 \times$ \\
\hline PK1 & U59 & $1.6 \times$ & $1.8 \times$ & $4.5 \times$ & $1.0 \times$ & $4.1 \times$ \\
\hline PK1 & G58 & $1.7 \times$ & $1.9 \times$ & $3.1 \times$ & $1.0 \times$ & $3.1 \times$ \\
\hline Loop in h2 & A34 & $4.9 \times$ & $1.0 \times$ & $1.0 \times$ & $1.0 \times$ & $1.0 \times$ \\
\hline Loop in h2 & A32 & $17.6 \times$ & $2.5 \times$ & $1.0 \times$ & $1.0 \times$ & $1.0 \times$ \\
\hline TLD & U17 & $10.0 \times$ & $2.2 \times$ & $1.0 \times$ & $1.0 \times$ & $1.0 \times$ \\
\hline TLD & U16 & $10.0 \times$ & $2.2 \times$ & $1.0 \times$ & $1.0 \times$ & $1.0 \times$ \\
\hline TLD & U10 & $2.4 \times$ & $1.5 \times$ & $14.1 \times$ & $1.0 \times$ & $10.7 \times$ \\
\hline TLD & $G 7^{\mathrm{C}}$ & $1.0 \times$ & $2.1 \times$ & $17.4 \times$ & $1.4 \times$ & $12.6 \times$ \\
\hline
\end{tabular}

'The lead(II) cleavages occur on the 3' side of the indicated nucleotides (e.g., C355 cleavage occurs in the phosphodiester backbone between C355 and G356).

${ }^{\mathrm{b}}$ Lead(II) cleavages were quantified as intensities of bands in Figure 5, using a PhosphorImager. The bands of lowest intensity, comparing the same nucleotide positions in all lanes, were set to unity $(1.0 \times)$. The intensities of the corresponding bands in other lanes are shown as relative increases. Significantly increased cleavages are indicated by boldface.

'Identification of the cleavage site at G7 is tentative in that it may, alternatively, be at A8. ribosomal complexes compared to naked tmRNA (Fig. 5C; Table 1), suggesting persistent interactions also of these latter tmRNA regions with the ribosome. The protection of A185 was, however, more pronounced in RCI, RCIII, and RCIV, than in RCII (Table 1).

A few other changes in reactivity to the lead(II) probe were confined to a subset of the ribosomal complexes. For instance, we observed lead(II)-induced cleavage at the double-stranded nucleotides G58 and U59 in h4 in RCII and IV (Figs. 2, 5B; Table 1), suggesting a selective destabilization of $\mathrm{h} 4$ in these two complexes. Moreover, the helical regions in PK4 became exposed to cleavage only in complex RCIII. Here, the putative double-stranded nucleotides A294 and A298 (Fig. 5D) were exposed to cleavage, suggesting breathing and/or general destabilization of this helix in RCIII. the first codon of the tmRNA ORF. The states of tmRNA in different complexes RCI-RCIV were verified by biochemical analysis (see Material and Methods for details).

tmRNA in the different ribosomal complexes was probed with lead(II) acetate. All complexes show strong protection from lead(II) cleavage in the TLD at positions U16-U17 (Fig. 5A) and G333-C335 (Fig. 5E), typical of the SmpB footprint on free tmRNA (Table 1). The protection was strongest in the RCI and RCII complexes and somewhat weaker in the RCIII and RCIV complexes. These results suggest that $\mathrm{SmpB}$ was attached to the same binding site on the tmRNA TLD (Fig. 3) in all four studied complexes and that the attachment was strongest in the RCI and RCII complexes. Cleavage at two nucleotides, U10 and G7 (see Table 1), on the $5^{\prime}$-proximal side of the D loop of tmRNA is much more efficient in RCII and RCIV than in other complexes or in naked tmRNA (Fig. 5A; Table 1). The increased lead cleavage activity at these two sites may reflect conformational changes in the elbow region of tmRNA upon formation of RCII or RCIV.

The lead(II) reactivity of the putative double-stranded nucleotide A34 in h2 and the single-stranded nucleotide A32 in the h2 internal loop (Fig. 2) was affected by the association of tmRNA with the ribosome. The protection of A32 and A34 was observed in all ribosomal complexes (Fig. 5A,E; Table 1), suggesting that this region of tmRNA was interacting with the ribosome during the studied steps of trans-translation. In addition, the accessibility of the single-stranded A185 in PK2 and the single-stranded A197 in PK3 (Fig. 2) to lead(II) cleavage was reduced in all

\section{DISCUSSION}

In this study we have used lead(II)-induced cleavage and DMS modifications to probe free tmRNA in the absence or presence of SmpB and to study ribosome-bound tmRNA at well-defined steps of trans-translation. For comparison, we have also probed tmRNA with lead(II) in wild type and SmpB-lacking E. coli cells. When SmpB binds to purified tmRNA in vitro it confers protection against lead(II) cleavage for nucleotides G333-C335 and U16-U17 in the TLD (Fig. 1). The same nucleotides are protected also in vivo in a wt but not in an isogenic SmpB-lacking strain (Fig. 1). These results suggest that, in vivo, a large fraction of tmRNA is bound to $\mathrm{SmpB}$, in line with recent results showing $\mathrm{SmpB}$ association with tmRNA during different stages of trans-translation in vivo (Shpanchenko et al. 2005).

The lead(II) cleavage patterns of tmRNA in the ribosomal complexes that we studied in vitro are similar (Fig. 5; Table 1), suggesting small changes in the secondary structure of tmRNA during the first trans-translation steps on the ribosome (see below). The absence of large changes of the tmRNA structure as its TLD passes through the tRNA binding sites on the ribosome may explain why the average in vivo lead(II) cleavage pattern is similar to the patterns observed in vitro (Fig. 1; Table 1).

SmpB confers protection against lead(II) cleavage in vivo, but not in vitro, also at nucleotides C66-U68 in PK1 (Fig. 1), not expected to be in direct contact with the protein (Fig. 3; Gutmann et al. 2003). Accordingly, this 


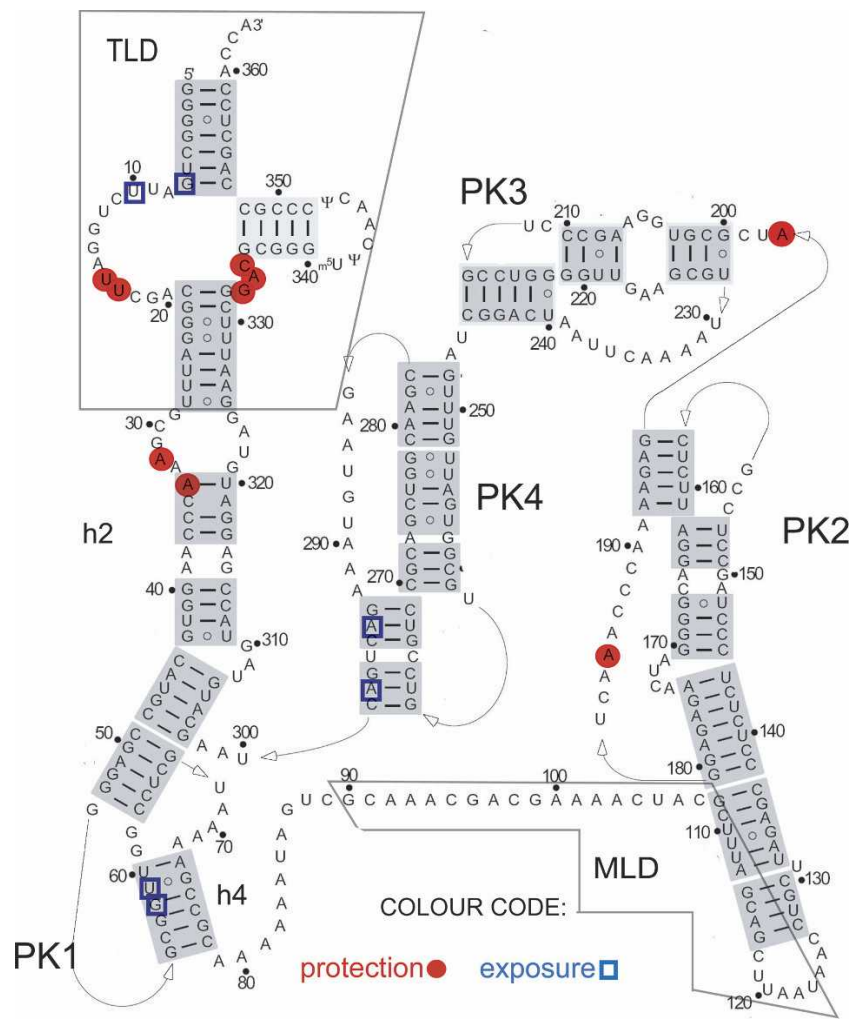

FIGURE 2. tmRNA secondary structure model with indicated nucleotide positions displaying changed susceptibility to lead(II) acetate. Specifically exposed or protected nucleotides identified in experiments in Figures 1 and 5 were mapped on the secondary structure of tmRNA Adapted with permission from Fig. 1A in Zwieb et al. (1999) (@) Oxford University Press). Empty blue squares mark the exposed nucleotides and filled red circles mark the protected nucleotides. The tRNA-like domain (TLD) and the mRNA-like domain (MLD) are indicated on the structure as well as pseudoknots 1-4 (PK1-PK4) and helices 2 and 4 (h2, h4). The lead(II) cleavages occur in the backbone of tmRNA on the $3^{\prime}$ side of marked nucleotides (e.g., cleavage at U10 occurs between U10 and C11).

protection could be due to different processing (Wower et al. 2004) and folding of tmRNA in vivo in the presence and absence of SmpB (Sundermeier et al. 2005).

The SmpB footprint is confined to a relatively small surface of tmRNA (Fig. 3). It includes nucleotides G333C335 and U16-U17 in the TLD seen to interact with SmpB in the SmpB.TLD crystal structure from A. aeolicus (Gutmann et al. 2003). The location of this footprint agrees with the suggestion that nucleotides $16-20$ and 334-335 in the acceptor stem of the TLD have functional interactions with SmpB (Barends et al. 2001; Wower et al. 2002; Metzinger et al. 2005) and the ribosome (Hanawa-Suetsugu et al. 2001). No additional SmpB protections were seen when SmpB was present at a large molar excess over tmRNA (Fig. 1), indicating that free tmRNA has one strong binding site for SmpB. This result is in line with the recent SmpB protection study (Nameki et al. 2005), but as many as three SmpB binding sites on tmRNA have been identified in another report (Metzinger et al. 2005). One possible explanation for this apparent discrepancy is that putative, weak binding sites outside the TLD of tmRNA (Metzinger et al. 2005) could have escaped detection in our lead-cleavage study.

Recent cryo-EM observations (Kaur et al. 2006) suggest that tmRNA in the preaccommodation state on the ribosome interacts with two copies of SmpB. This conclusion is perfectly in line with earlier biochemical (Hallier et al. 2004) and footprinting (Ivanova et al. 2005a) studies. One SmpB molecule (SmpB-1) is situated adjacent to the TLD of tmRNA near the GTPase-associated center (GAC) of the $50 \mathrm{~S}$ subunit, while the other (SmpB-2) is positioned in the decoding center of the $30 \mathrm{~S}$ subunit (Kaur et al. 2006). The positioning of SmpB-1 near helices H43 and H44 of the 50S subunit in a very recent cryo-EM structure (Kaur et al. 2006) deviates from the positioning of SmpB-1 in a previous study by the same group (Valle et al. 2003), but agrees well with footprinting results (Ivanova et al. 2005a). SmpB-2 is tightly bound to preaccommodation tmRNA by interactions with the D loop of the TLD (Kaur et al. 2006), as previously seen in the crystal complex of SmpB and the TLD (Fig. 3; Gutmann et al. 2003). SmpB-2 remained in the same position in the A site of the $30 \mathrm{~S}$ subunit after the release of EF-Tu from the ribosome and tmRNA accommodation, while no firm conclusion was reached regarding the positioning and even the presence of SmpB-1 in this post-accommodations complex (Kaur et al. 2006). The

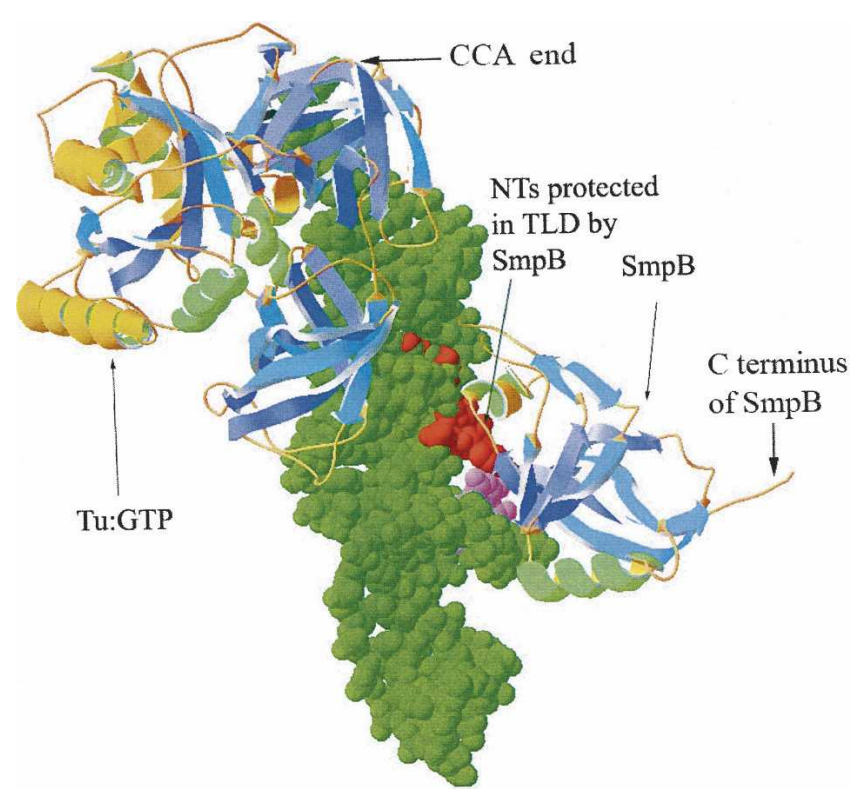

FIGURE 3. A 3D model of a complex between SmpB, EF-Tu•GTP, and tRNA-like domain (TLD) of tmRNA (green). Nucleotides of TLD protected from lead(II) cleavage are shown in red (G333-C335) and pink (U16, U17). The model was derived by combining the crystal structure of the SmpB $\cdot T L D$ complex (PDB file 1V6P) with a crystal structure of EF-Tu•GTP in complex with Phe-tRNA ${ }^{\text {Phe }}$ (PDB file 1TTT) by aligning the T loop of tRNA and TLD. 


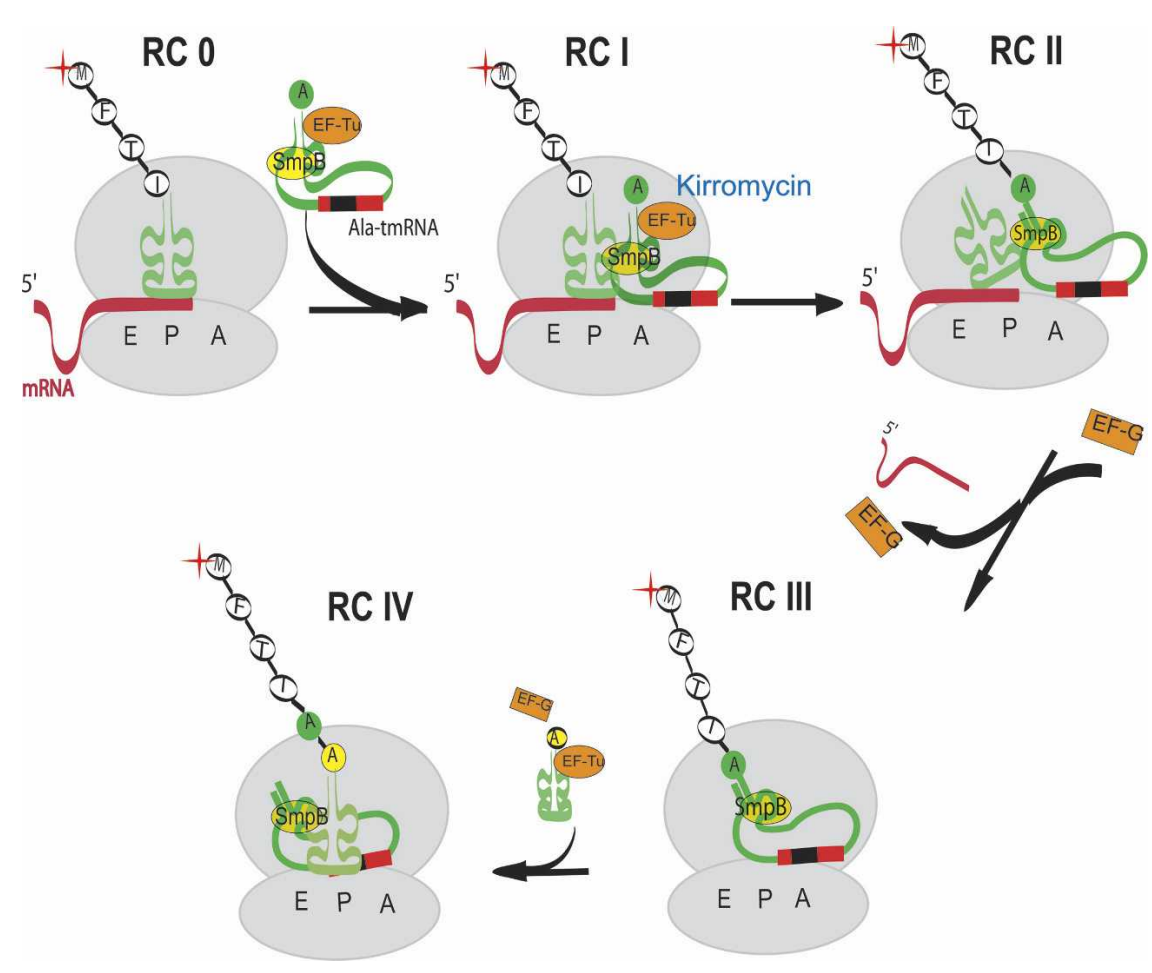

FIGURE 4. Trans-translation steps. Ribosomal complexes (RCs) were prepared and purified as described in Materials and Methods. (RCO) ribosomes contain MFTI-tRNA ${ }^{\text {Ile }}$ in the P site and an empty A site; (RCI) ribosomes contain MFTI-tRNA ${ }^{\text {Ile }}$ in the P site and AlatmRNA $\bullet \mathrm{SmpB} \bullet \mathrm{Tu} \cdot \mathrm{GDP}$ complex stalled by the antibiotic kirromycin in the A site; (RCII) ribosomes contain deacylated tRNA ${ }^{\text {Ile }}$ in the P site and MFTIA-tmRNA in the A site; (RCIII) ribosomes contain deacylated $\mathrm{RNA}^{\text {Ile }}$ in the E site, MFTIA-tmRNA in the P site, and the resume codon of tmRNAs internal ORF in the A site; (RCIV) ribosomes contain deacylated tmRNA in the E site, MFTIAA-tRNA ${ }^{\text {Ala }}$ in the P site and an empty A site containing the second codon of tmRNA's internal ORF.

SmpB-2 positioning derived by cryo-EM is also in line with our lead(II)-cleavage data (Table 1), suggesting that SmpB2 remains tmRNA bound both in the pre- and postaccommodation complexes. Moreover, the characteristic SmpB-2 footprint on tmRNA is present also in posttranslocation complexes RCIII and RCIV (Table 1), suggesting that SmpB-2 remains attached to tmRNA as it moves from the $\mathrm{A}$ to the $\mathrm{P}$ to the $\mathrm{E}$ site on the ribosome. SmpB-2 may mimic the anticodon loop of tRNAs that is missing in tmRNA (Kaur et al. 2006), thereby facilitating the positioning of tmRNA in the $\mathrm{P}$ or $\mathrm{E}$ site and the translocation of the TLD from ribosome site to ribosome site as previously suggested with support from footprinting data (Ivanova et al. 2005a). Interestingly, nucleotides G7 and $\mathrm{U} 10$ on the $5^{\prime}$ side of the D loop of tmRNA are much more exposed to lead II cleavage in the post-accommodation complex RCII and in the twice-translocated complex RCIV than in naked tmRNA and other tmRNA complexes (Fig. 5A; Table 1). This suggests change in the conformation of the elbow region of tmRNA as its CCA end accommodates in the A site of the 50S subunit. A similar conformational change may also occur in the tmRNA as its
CCA end moves into the E site of the 50 subunit in complex RCIV.

The increased protection of nucleotides A185 in PK2 and A197 in PK3 of tmRNA (Fig. 2) in all ribosomal complexes as compared to free tmRNA suggests that PK2 and PK3 remain intact and interact with the ribosome as the TLD of tmRNA moves from site to site on the ribosome. Interestingly, only a small number of nucleotides in putative double-stranded regions of tmRNA become exposed to lead(II) cleavage after tmRNA accommodation and/or translocation by EF-G (Fig. 5; Table $1)$, strongly suggesting that tmRNA retains its overall secondary structure during the first steps of trans-translation. Similar conclusions regarding conservation of the secondary structure of tmRNA during trans-translation have been drawn from other types of experiments (Ivanov et al. 2002; Shpanchenko et al. 2005).

It can be noted that while our data suggest the secondary structure of tmRNA to remain essentially unaltered as the TLD moves from complex RCI to RCIV (Table 1), it was suggested from the cryo-EM data that PK4, and to some extent also PK2 and PK3, become unwound or in other ways more mobile as tmRNA accommodates in the A site (Kaur et al. 2006). One reason for this apparent contradiction could be the low occupancy of tmRNA in the postaccommodated state in this cryo-EM study, which is expected to reduce the reliability of the reconstruction. In addition, the putative unwinding of pseudo-knot structures PK2-PK4 (Kaur et al. 2006) would require a significant input of free energy from a yet unidentified source. The observation that replacement of the stop codons at the end of the ORF of tmRNA with sense codons results in the synthesis of abnormally long-tag peptides (Wower et al. 2005) shows that unwinding of the tmRNA secondary in the PK2-PK4 can take place. However, in this latter case the free energy for unwinding is provided by protein elongation, which supports the present and previous conclusions (Ivanov et al. 2002; Shpanchenko et al. 2005) that the putative unwinding of tmRNA takes place at a much later stage of trans-translation than tmRNA accommodation in the A site.

Nucleotides A32 and A34 were protected from lead(II) cleavage in all ribosomal complexes (Fig. 5; Table 1). Their protection in RCI and RCII may be due to interactions with ribosomal protein S1 (Bordeau and Felden 2002). However, in the further downstream trans-translation complexes 


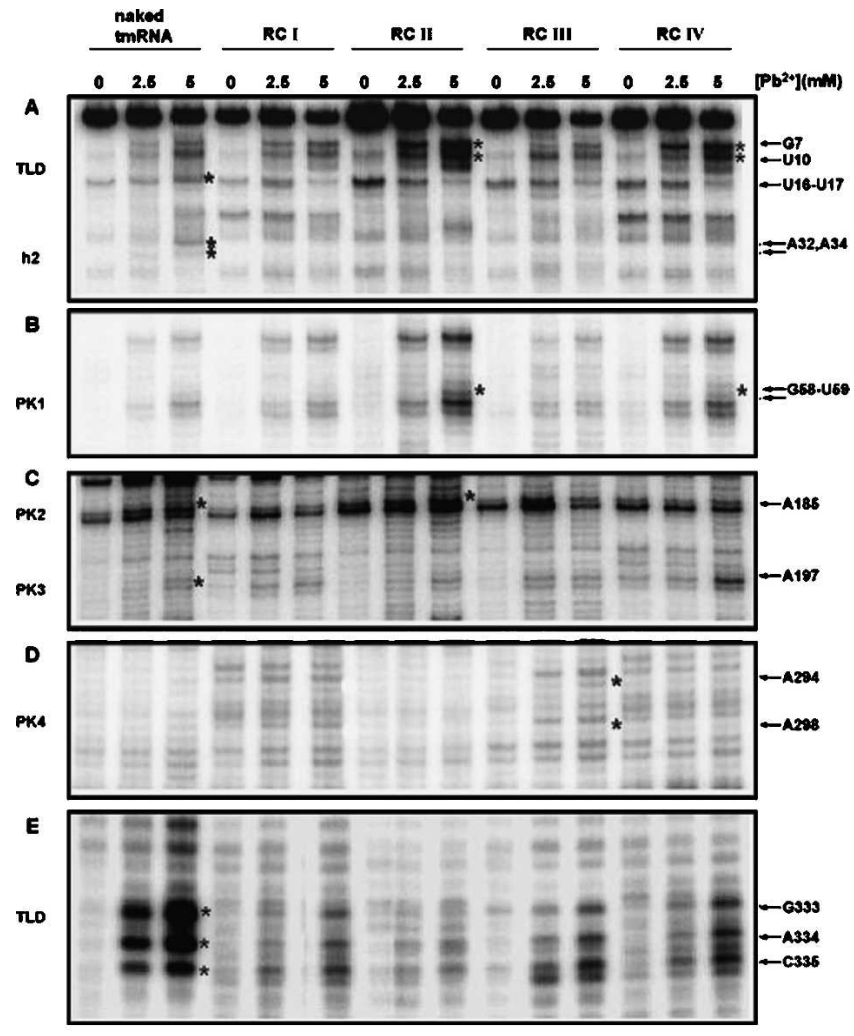

FIGURE 5. Lead(II) acetate cleavages on tmRNA in different ribosomal complexes. Lead(II) cleavage analyses were performed on four different ribosomal complexes (RCI-IV). Primer extensions of naked tmRNA were run on the same gels for comparison. Three different radiolabeled primers (GW-TM2, GW-TM3, and GW-TM5) were used for primer extension analysis, and the lead(II) concentrations for cleavage were 2.5 and $5 \mathrm{mM}$. Nucleotides U16-U17 (A) and G333C335 (E), which are protected in the RCs due to interaction with $\mathrm{SmpB}$, are indicated to the right of the autoradiograph. Nucleotides G7 and U10 (A) as well as G58 and U59 (B), which are uniquely cleaved in complexes RCII and RC IV, are marked. A few more nucleotide positions (A185, A197, A294, and A298) (C,D), which show differences in cleavage accessibility between naked tmRNA and tmRNA in different RCs, are also marked. Nucleotides A32 and A34 $(A)$, which are protected in all RCs, are indicated as well. A detailed evaluation of cleavages is shown in Table 1. Asterisks in the figure mark the significantly increased cleavages that are in boldface in Table 1.

RCIII and RCIV (Fig. 4), A32 and A34 are, we suggest, in the intersubunit space, in which case they are likely to be protected by other parts of the ribosome than S1 (Ivanov et al. 2002).

The limits of resolution of the probing experiments presented here and incomplete knowledge of the detailed interactions of tmRNA with other key components in the individual trans-translation steps make it difficult to assign specific roles to the nucleotides that exhibit changed accessibility to probing in particular ribosomal complexes (Fig. 5; Table 1). At the same time, the observed changes in the protection pattern must reflect the movement of tmRNA through the ribosome during trans-translation. Footprinting studies of ribosomal RNA are currently performed on the same ribosomal complexes RCI-RCIV to obtain complementary information on the tmRNAribosome interactions. Additionally, cryoelectron microscopy studies of the same complexes that are underway are expected to provide more and vital information on the conformation of tmRNA and the interactions of tmRNA with the ribosome at different stages of trans-translation.

\section{MATERIALS AND METHODS}

\section{Chemicals and buffers}

Nucleoside triphosphates, RNAguard (porcine), and radioisotopes were from Amersham Biosciences; phosphoenol pyruvate (PEP), putrescine, spermidine, myokinase (MK), and nonradioactive amino acids were from Sigma; pyruvate kinase (PK) was from Boehringer; inorganic pyrophosphatase was from New England Biolabs; and other chemicals were from Merck. Polymix buffer (Jelenc and Kurland 1979), contained at working strength $5 \mathrm{mM}$ $\mathrm{Mg}(\mathrm{OAc})_{2}, 0.5 \mathrm{mM} \mathrm{CaCl}_{2}, 5 \mathrm{mM} \mathrm{NH}_{4} \mathrm{Cl}, 95 \mathrm{mM} \mathrm{KCl}, 8 \mathrm{mM}$ putrescine, $1 \mathrm{mM}$ spermidine, $5 \mathrm{mM}$ potassium phosphate $(\mathrm{pH}$ 7.3), and $1 \mathrm{mM}$ DTE. TMK buffer contained at final concentration $50 \mathrm{mM}$ TrisOAc (pH 7.5), $50 \mathrm{mM} \mathrm{KOAc}$, and $10 \mathrm{mM} \mathrm{Mg}(\mathrm{OAc})_{2}$.

\section{Purification of overexpressed his-tagged SmpB}

His-tagged SmpB was purified from E. coli strain BL21(DE3)/ pet-21a-SmpB as described by Hallier et al. (2004) and Ivanova et al. (2004).

\section{In vitro transcription and purification of tmRNA}

tmRNA was prepared by in vitro T7 transcription of the PCR amplified ssrA gene as described by Hallier et al. (2004). The forward PCR amplification primer, 5'-GGTACCGAAATTAATAC GACTCACTATAGGGGCTGATTCTGGATTCGACGG, introduced a T7 promoter into the PCR product, and the 5'-TGGTGGAGCT GGCGGGAGTTGAACC oligo was used as a reverse primer. The transcription reaction was carried out according to Pokrovskaya and Gurevich (1994) in $4 \mathrm{~mL}$ of $200 \mathrm{mM}$ HEPES (pH 7.5), $30 \mathrm{mM}$ $\mathrm{MgCl}_{2}$, $30 \mathrm{mM}$ DTE, $2 \mathrm{mM}$ spermidine, $7 \mathrm{mM}$ ATP, UTP, CTP, and GTP, 1 nmol PCR DNA, $0.3 \mathrm{U} / \mu \mathrm{L}$ RNA-guard, $2 \mathrm{U} / \mathrm{mL}$ inorganic pyrophosphatase, and $0.01 \mathrm{mg} / \mathrm{mL}$ T7 RNA polymerase. After a $4 \mathrm{~h}$ incubation, the reaction was extracted twice with phenol/chloroform and ethanol precipitated. The precipitate was collected by centrifugation for $30 \mathrm{~min}$ at $21,000 \mathrm{~g}$, dissolved in $0.5 \mathrm{~mL}$ of water, and applied onto a $50 \mathrm{~mL}$ Sephacryl S300 column (Amersham) equilibrated with water. Partially purified tmRNA was concentrated by ultrafiltration in Amicon spin concentrators (cutoff $10 \mathrm{kDa}$ ) and further purified by denaturing 5\% PAGE (5\% acrylamide/bisacrylamide; $7 \mathrm{M}$ urea, in $1 \times \mathrm{TBE}-90 \mathrm{mM}$ Tris $(\mathrm{pH}$ 8), $90 \mathrm{mM}$ borate, $2 \mathrm{mM}$ EDTA). The full-length tmRNA band was visualized by UV shadowing and excised from the gel. tmRNA was extracted by electroelution for $1 \mathrm{~h}$ in a BIORAD Electro-Eluter (Model 422) as described in the manual.

\section{Alanylation of tmRNA}

tmRNA $(5 \mu \mathrm{M})$ was aminoacylated with Ala in polymix buffer containing $7.5 \mathrm{mM} \mathrm{Mg}(\mathrm{OAc})_{2}, 1.5 \mathrm{mM}$ ATP, $15 \mathrm{mM}$ PEP, $2 \mathrm{mM}$ 
GTP, $1 \mathrm{mM}$ Ala (or $0.1 \mathrm{mM}\left[{ }^{3} \mathrm{H}\right]$ or $\left.\left[{ }^{14} \mathrm{C}\right] \mathrm{Ala}\right), 0.45 \mathrm{U} / \mu \mathrm{L}$ alanyltRNA-synthetase, and $0.34 \mathrm{U} / \mu \mathrm{L}$ RNAguard. After $15 \mathrm{~min}$ incubation, Ala-tmRNA was extracted with phenol/chloroform saturated with $0.2 \mathrm{M} \mathrm{NaOAc}$ at $\mathrm{pH} 5$ and precipitated with ethanol. The precipitate was collected by centrifugation, dissolved in water, and stored in aliquots at $-80^{\circ} \mathrm{C}$. The final extent of tmRNA alanylation was $30 \%$, as judged from the aminoacylation reaction performed with $\left[{ }^{14} \mathrm{C}\right] \mathrm{Ala}(250 \mathrm{cpm} / \mathrm{pmol})$ or $\left[{ }^{3} \mathrm{H}\right] \mathrm{Ala}$ (400 cpm/pmol).

\section{Purification of tmRNA.SmpB, tmRNA.EF-Tu, and tmRNA.SmpB-EF-Tu.GTP complexes}

tmRNA $\cdot S m p B$ complexes were prepared by incubating tmRNA with a fivefold molar excess of his-tagged $\mathrm{SmpB}$ for $5 \mathrm{~min}$ at $37^{\circ} \mathrm{C}$. For preparation of Ala-tmRNA $\bullet \mathrm{SmpB} \cdot \mathrm{EF}-\mathrm{Tu} \cdot \mathrm{GTP}$ complexes, both His-tagged SmpB and His-tagged EF-Tu were added to the tmRNA alanylation reaction mix. After 15 min incubation, the complexes were purified on a $\mathrm{Ni}^{2+}$-nitrilotriacetic acid (NTA) column (Qiagen) as described by Barends et al. (2001). The reaction mix was loaded onto a $1 \mathrm{~mL} \mathrm{Ni}^{2+}$-NTA column (Qiagen). The column was washed with buffer A (50 mM K-phosphate at $\mathrm{pH}$ 6.5, $250 \mathrm{mM} \mathrm{KCl,} 1 \mathrm{mM} \beta$-mercaptoethanol, $2 \mathrm{mM} \mathrm{MgCl}_{2}$, $15 \mathrm{mM}$ imidazole), after which the bound proteins and tmRNA were eluted with $200 \mathrm{mM}$ imidazole in buffer A. Fractions containing $\mathrm{SmpB} / \mathrm{EF}-\mathrm{Tu} \cdot \mathrm{GTP} \bullet A l a-t m R N A$ were concentrated to $200 \mu \mathrm{L}$ by ultrafiltration as described above and stored in aliquots at $-80^{\circ} \mathrm{C}$. Alternatively, Ala-tmRNA $\bullet \mathrm{SmpB} \cdot \mathrm{EF}-\mathrm{Tu} \cdot \mathrm{GTP}$ complexes were prepared from Ni-NTA purified Ala-tmRNA $\bullet E F-T u \cdot G T P$ complexes by adding a fivefold molar excess of His-tagged SmpB for $5 \mathrm{~min}$ at $37^{\circ} \mathrm{C}$.

\section{In vitro translation and isolation of stalled ribosomal complexes (RCs), containing MFTI-tRNA ${ }^{\text {Ile }}$}

Ribosomal complexes (RC0; see Fig. 4) were prepared by translation of a short ORF encoding the MFTI tetrapeptide in the absence of release factors (Freistroffer et al. 1997). The mRNA sequence was: GGGAAGCUGAACGAGAAACGUAAAAUGUUU ACGAUUUAGUAC (Shine and Dalgarno and coding sequence underlined; stop codon in bold). The components of translation reaction were prepared as described by Ivanova et al. (2005b). The prepared RC0, containing fMet-Phe-Thr-Ile-tRNA ${ }^{\text {Ile }}$ (MFTI-tRNA ${ }^{\text {Ile }}$ ) in the $\mathrm{P}$ site with $\left[{ }^{3} \mathrm{H}\right]$-labeled fMet or Ile, was further purified from other components of the translation mixture by gel filtration through a Sephacryl S300 column (Freistroffer et al. 1997). The MFTI content of RC0 and its reactivity in trans-transfer (MFTIA formation) were quantified by HPLC as described by Hallier et al. (2004).

\section{In vitro trans-translation and purification of ribosomal rescue complexes containing tmRNA}

The ribosomal rescue complexes, arrested at different stages of trans-translation, were prepared as follows: RCI was prepared by mixing $1 \mu \mathrm{M}$ RC0 with $1 \mu \mathrm{M}$ Ala-tmRNA $\bullet$ SmpB $\bullet E F-T u \bullet\left[{ }^{3} \mathrm{H}\right] \mathrm{GTP}$ complex purified on a Ni-NTA column from nonactive tmRNA as described above in the presence of $100 \mu \mathrm{M}$ kirromycin and $100 \mu \mathrm{M}\left[{ }^{3} \mathrm{H}\right] \mathrm{GTP}$ in the reaction mix. $\left[{ }^{3} \mathrm{H}\right] \mathrm{GTP}$ was used to monitor the amount of EF-Tu•GDP•kirromycin retained on the ribosome. The reaction was performed at $37^{\circ} \mathrm{C}$ for $3 \mathrm{~min}$, and the resulting RCI was further purified from unbound

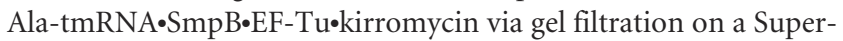
dex S-400 column in TMK buffer. After purification the RCI sample contained neither ribosomes bound to deacylated tmRNA nor free tmRNA. The purified RCI was concentrated to $0.3 \mu \mathrm{M}$ for the lead(II) cleavage assays using Microcon YM-100 (cutoff $100 \mathrm{kDa}$ ). RCII was prepared in the same way as RCI but in the absence of kirromycin so that EF-Tu was able to leave the ribosome and the transfer of the tetrapeptide to Ala-tmRNA could occur. To confirm the trans-transfer, aliquots of the reaction mix were quenched in formic acid (FA) at $17 \%$ final concentration and the extent of fMet-Phe-Thr-Ile-Ala formation was quantified by HPLC from the ratio of the AMFTIA peak to the sum of the fMFTI and fMFTIA peaks as described by Hallier et al. (2004). One hundred percent of the tetrapeptide was transferred to Ala-tmRNA in the case of RCII. RCIII was prepared from RCII by adding EF-G (final concentration $1 \mu \mathrm{M}$ ), which promoted the translocation of MFTIA-tmRNA to the ribosomal $\mathrm{P}$ site. The extent of translocation was quantified by puromycin reaction (Ivanova et al. 2005b). Puromycin was added at $400 \mu \mathrm{M}$ final concentration and the reaction was stopped after different times by FA added to $5 \%$ final concentration. The fraction of the MFTIA pentapeptide that could be released from ribosomal complexes corresponded to the extent of MFTIA-tmRNA translocation (Ivanova et al. 2005b). After $5 \mathrm{sec}, 100 \%$ of MFTIA could be released by puromycin. The puromycin reactivity of RCII was measured as a negative control, and no activity was found. RCIV was obtained from RCIII by adding a $2 \times$ molar excess of purified $\left[{ }^{14} \mathrm{C}\right]$ Ala-tRNA ${ }^{\mathrm{Ala}}$ in the presence of $1 \mu \mathrm{M}$ EF-G, $5 \mu \mathrm{M}$ EF-Tu•GTP, and $100 \mu \mathrm{M}$ GTP. The procedure for alanylation and purification of $\left[{ }^{14} \mathrm{C}\right] \mathrm{Ala}-\mathrm{tRNA}{ }^{\mathrm{Ala}}$ was the same as for AlatmRNA described above.

\section{In vivo lead(II) acetate cleavage of tmRNA}

The protocol used here was essentially as described by Lindell et al. (2002). E. coli cells (isogenic wt and $\Delta s m p B$ strains) were grown at $37^{\circ} \mathrm{C}$ in $\mathrm{L}$ Broth (LB). Overnight cultures were diluted $100 \times$ in LB and grown to mid-log phase $\left(\mathrm{OD}_{600}=0.5\right)$. Immediately before treatment, lead(II) solutions were prepared by mixing $3 \mathrm{vol}$ of $1 \mathrm{M}$ lead(II) acetate (Merck) in $\mathrm{H}_{2} \mathrm{O}$ with 1 vol of prewarmed $4 \times$ concentrated LB, added to cultures to give a final lead(II) concentration of $100 \mathrm{mM}$, and incubated at $37^{\circ} \mathrm{C}$ with vigorous shaking for $7 \mathrm{~min}$. Reactions were stopped by addition of a 1.5fold molar excess of EDTA on ice. Total RNA was extracted by the hot phenol method (Blomberg et al. 1990), and RNA samples were further treated with DNase I (Amersham Biosciences).

Lead(II) cleavages were identified by primer extension. The $5^{\prime}-$ $\left[{ }^{32} \mathrm{P}\right]$-end-labeled primers, GW-TM2 (5'-GCTTGCCAGCTGCG GA), GW-TM3 (5'-GAGAGAGGGCTCTAAGCA), and GW-TM5 (5'-TGGTGGAGCTGGCGGGA), were annealed to $10 \mu \mathrm{g}$ of total RNA (incubations: $90^{\circ} \mathrm{C}$ for $1 \mathrm{~min}, 1 \mathrm{~min}$ on ice, and $5 \mathrm{~min}$ at $20^{\circ} \mathrm{C}$ ), and primer extension was performed at $45^{\circ} \mathrm{C}$ for $30 \mathrm{~min}$ using 200 units of Superscript II (Invitrogen) in a volume of 15 $\mu \mathrm{L}$, containing $50 \mathrm{mM}$ Tris- $\mathrm{HCl}(\mathrm{pH} 8.5), 6 \mathrm{mM} \mathrm{MgCl}_{2}, 40 \mathrm{mM}$ $\mathrm{KCl}$, and $1.0 \mathrm{mM}$ dNTP each. Reactions were terminated with $20 \mu \mathrm{L}$ of stop buffer ( $50 \mathrm{mM}$ Tris- $\mathrm{HCl}$ at $\mathrm{pH} 7.5,0.1 \%$ SDS $)$ and $3.5 \mu \mathrm{L}$ of $3 \mathrm{M} \mathrm{KOH}$. After $3 \mathrm{~min}$ at $90^{\circ} \mathrm{C}$, and subsequently $3 \mathrm{~h}$ at $37^{\circ} \mathrm{C}, 6 \mu \mathrm{L}$ of $3 \mathrm{M}$ acetic acid were added, and the cDNA was 
precipitated with ethanol. Reverse transcription products were separated on $8 \%$ sequencing gels. Cleavage positions were identified by comparison to sequencing reactions using the same $5^{\prime}$ end-labeled primers; templates were generated by colony PCR using the following primer pair: TMR-A (5'-GGTCATGGCGCT CATAAATC) and TMR-B-T7 (5'-GAAATTAATACGACTCAC TATAGGTGGAGCTGGCGGGA). The circumvent thermal cycle dideoxy DNA sequencing kit (New England Biolabs) was used according to the manufacturer's protocol.

\section{Analysis of primer extension data}

Overall, several classes of cleavages were observed: (1) lead(II)induced cleavages/protections, which can be subdivided into (a) protections attributable to SmpB binding, (b) changed susceptibility to lead(II) in all complexes as compared to free tmRNA, (c) changed susceptibility to lead(II) in some complexes, and (d) changed susceptibility to lead(II) in one particular complex.

(2) Lead-independent cleavages, which can be subdivided into (a) ubiquitous apparent cleavages, representing fragile sites in tmRNA or cleavage-independent reverse transcription stops; (b) cleavages absent in tmRNA off the ribosome; and (c) stage-specific cleavages-most likely originating from ribosome or ribosomebound endonucleolytic activities. Increased/decreased accessibility of nucleotides to lead(II)-induced cleavage was analyzed on a PhosphorImager. Quantification of each cleavage was scored manually.

\section{In vitro lead(II) acetate cleavage of tmRNA}

One picomole of tmRNA:SmpB complexes purified on a Ni-NTA column or 1 pmol in vitro transcribed tmRNA with or without $\mathrm{SmpB}$ (concentration indicated in figures) was preincubated for 10 $\mathrm{min}$ at $37^{\circ} \mathrm{C}$ in $50 \mathrm{mM}$ Tris-OAc ( $\mathrm{pH}$ 7.6), $50 \mathrm{mM} \mathrm{KAc}, 5 \mathrm{mM}$ $\mathrm{Mg}(\mathrm{OAc})_{2}$, and $1.0 \mu \mathrm{M}$ CopT RNA. A 345-nt RNA (CopT) was added as a carrier instead of tRNA to achieve less than one cleavage per molecule, thereby avoiding secondary cuts. Cleavage was initiated by the addition of lead(II) acetate ( $5 \mathrm{mM}$ concentration in $25 \mu \mathrm{L}$ ). After $5 \mathrm{~min}$ at $37^{\circ} \mathrm{C}$, the reaction was stopped by the addition of $5 \mu \mathrm{L} 0.1 \mathrm{M}$ EDTA and $75 \mu \mathrm{L}$ 99\% ethanol. Precipitated RNA was vacuum dried and dissolved in water prior to analysis on PAGE or primer extension. Lead(II) cleavage analysis on $3.3 \mathrm{pmol}$ of the five different RCs was performed as described previously, but the final lead(II) concentration was 2.5 or $5 \mathrm{mM}$.

\section{In vitro DMS modification of tmRNA}

Ten picomoles of tmRNA $\bullet$ SmpB complexes purified on a Ni-NTA column or $10 \mathrm{pmol}$ in vitro transcribed tmRNA with or without SmpB protein were preincubated for $5 \mathrm{~min}$ at $37^{\circ} \mathrm{C}$ in $50 \mathrm{mM}$ Tris-OAc (pH 7.6), $50 \mathrm{mM} \mathrm{KOAc}$, and $5 \mathrm{mM} \mathrm{Mg}(\mathrm{OAc})_{2}$. Reactions were left on ice for $10 \mathrm{~min}$ before DMS (Acros Organics; diluted 1:15 in ethanol) was added to a concentration of $25 \mathrm{mM}$ (final volume $60 \mu \mathrm{L}$ ). DMS methylation of unpaired A:s at the $\mathrm{N}-1$ position and unpaired C:s at the $\mathrm{N}-3$ position was allowed to proceed for $5 \mathrm{~min}$ at $37^{\circ} \mathrm{C}$. Control samples containing no modifying reagent were incubated in parallel. Reactions were precipitated with 0.1 vol $3 \mathrm{M} \mathrm{NH}_{4} \mathrm{Ac}(\mathrm{pH} 5.2)$ and 2.5 vol EtOH. Precipitated RNA was extracted from protein by phenol extractions before the samples were analyzed by primer extension.

\section{ACKNOWLEDGMENTS}

This work was supported by the Swedish Research Council (M.E and E.G.H.W.) and the European Commission (EU-STREP FOSRAK) (E.G.H.W.).

Received December 28, 2006; accepted February 19, 2007.

\section{REFERENCES}

Andersen, E.S., Rosenblad, M.A., Larsen, N., Westergaard, J.C., Burks, J., Wower, I.K., Wower, J., Gorodkin, J., Samuelsson, T., and Zwieb, C. 2006. The tmRDB and SRPDB resources. Nucleic Acids Res. 34: 163-168.

Atkins, J.F. and Gesteland, R.F. 1996. A case for trans-translation. Nature 379: 769-771.

Barends, S., Wower, J., and Kraal, B. 2000. Kinetic parameters for tmRNA binding to alanyl-tRNA synthetase and elongation factor Tu from Escherichia coli. Biochemistry 39: 2652-2658.

Barends, S., Karzai, A.W., Sauer, R.T., Wower, J., and Kraal, B. 2001. Simultaneous and functional binding of SmpB and EF-Tu.GTP to the alanyl acceptor arm of tmRNA. J. Mol. Biol. 314: 9-21.

Blomberg, P., Wagner, E.G.H., and Nordström, K. 1990. Control of replication of plasmid R1: The duplex between the antisense RNA, CopA, and its target, CopT, is processed specifically in vivo and in vitro by RNase III. EMBO J. 9: 2331-2340.

Bordeau, V. and Felden, B. 2002. Ribosomal protein S1 induces a conformational change of tmRNA; more than one protein S1 per molecule of tmRNA. Biochimie 84: 723-729.

Felden, B., Himeno, H., Muto, A., McCutcheon, J.P., Atkins, J.F., and Gesteland, R.F. 1997. Probing the structure of the Escherichia coli 10Sa RNA (tmRNA). RNA 3: 89-103.

Felden, B., Massire, C., Westhof, E., Atkins, J.F., and Gesteland, R.F. 2001. Phylogenetic analysis of tmRNA genes within a bacterial subgroup reveals a specific structural signature. Nucleic Acids Res. 29: 1602-1607.

Freistroffer, D.V., Pavlov, M.Y., MacDougall, J., Buckingham, R.H., and Ehrenberg, M. 1997. Release factor RF3 in E. coli accelerates the dissociation of release factors RF1 and RF2 from the ribosome in a GTP-dependent manner. EMBO J. 16: 4126-4133.

Gaudin, C., Zhou, X., Williams, K.P., and Felden, B. 2002. Two-piece tmRNA in cyanobacteria and its structural analysis. Nucleic Acids Res. 30: 2018-2024.

Gornicki, P., Baudin, F., Romby, P., Wiewiorowski, M., Kryzosiak, W., Ebel, J.P., Ehresmann, C., and Ehresmann, B. 1989. Use of lead(II) to probe the structure of large RNA's. Conformation of the $3^{\prime}$ terminal domain of E. coli $16 \mathrm{~S}$ rRNA and its involvement in building the tRNA binding sites. J. Biomol. Struct. Dyn. 6: 971-984.

Gutmann, S., Haebel, P.W., Metzinger, L., Sutter, M., Felden, B., and Ban, N. 2003. Crystal structure of the transfer-RNA domain of transfer-messenger RNA in complex with SmpB. Nature 424: 699-703.

Hallier, M., Ivanova, N., Rametti, A., Pavlov, M., Ehrenberg, M., and Felden, B. 2004. Pre-binding of small protein B to a stalled ribosome triggers trans-translation. J. Biol. Chem. 279: 2597825985.

Hanawa-Suetsugu, K., Bordeau, V., Himeno, H., Muto, A., and Felden, B. 2001. Importance of the conserved nucleotides around the tRNA-like structure of Escherichia coli transfer-messenger RNA for protein tagging. Nucleic Acids Res. 29: 4663-4673.

Hanawa-Suetsugu, K., Takagi, M., Inokuchi, H., Himeno, H., and Muto, A. 2002. SmpB functions in various steps of trans-translation. Nucleic Acids Res. 30: 1620-1629.

Hickerson, R.P., Watkins-Sims, C.D., Burrows, C.J., Atkins, J.F., Gesteland, R.F., and Felden, B. 1998. A nickel complex cleaves 


\section{Ivanova et al.}

uridine in folded RNA structures: Application to E. coli tmRNA and related engineered molecules. J. Mol. Biol. 279: 577-587.

Ivanov, P.V., Zvereva, M.I., Shpanchenko, O.V., Dontsova, O.A., Bogdanov, A.A., Aglyamova, G.V., Lim, V.I., Teraoka, Y., and Nierhaus, K.H. 2002. How does tmRNA move through the ribosome? FEBS Lett. 514: 55-59.

Ivanova, N., Pavlov, M.Y., Felden, B., and Ehrenberg, M. 2004. Ribosome rescue by tmRNA requires truncated mRNAs. J. Mol. Biol. 338: 33-41.

Ivanova, N., Pavlov, M.Y., Bouakaz, E., Ehrenberg, M., and Schiavone, L.H. 2005a. Mapping the interaction of SmpB with ribosomes by footprinting of ribosomal RNA. Nucleic Acids Res. 33: 3529-3539.

Ivanova, N., Pavlov, M.Y., and Ehrenberg, M. 2005b. tmRNA-induced release of messenger RNA from stalled ribosomes. J. Mol. Biol. 350: 897-905.

Jelenc, P.C. and Kurland, C.G. 1979. Nucleoside triphosphate regeneration decreases the frequency of translation errors. Proc. Natl. Acad. Sci. 76: 3174-3178.

Karzai, A.W., Susskind, M.M., and Sauer, R.T. 1999. SmpB, a unique RNA-binding protein essential for the peptide-tagging activity of SsrA (tmRNA). EMBO J. 18: 3793-3799.

Kaur, S., Gillet, R., Li, W., Gursky, R., and Frank, J. 2006. Cryo-EM visualization of transfer messenger RNA with two $\mathrm{SmpBs}$ in a stalled ribosome. Proc. Natl. Acad. Sci. 103: 16484-16489.

Keiler, K.C. and Sauer, R.T. 1996. Sequence determinants of Cterminal substrate recognition by the Tsp protease. J. Biol. Chem. 271: 2589-2593.

Keiler, K.C., Shapiro, L., and Williams, K.P. 2000. tmRNAs that encode proteolysis-inducing tags are found in all known bacterial genomes: A two-piece tmRNA functions in Caulobacter. Proc. Natl. Acad. Sci. 97: 7778-7783.

Knudsen, B., Wower, J., Zwieb, C., and Gorodkin, J. 2001. tmRDB (tmRNA database). Nucleic Acids Res. 29: 171-172.

Komine, Y., Kitabatake, M., Yokogawa, T., Nishikawa, K., and Inokuchi, H. 1994. A tRNA-like structure is present in 10Sa RNA, a small stable RNA from Escherichia coli. Proc. Natl. Acad. Sci. 91: 9223-9227.

Lindell, M., Romby, P., and Wagner, E.G.H. 2002. Lead(II) as a probe for investigating RNA structure in vivo. RNA 8: 534-541.

Metzinger, L., Hallier, M., and Felden, B. 2005. Independent binding sites of small protein B onto transfer-messenger RNA during trans-translation. Nucleic Acids Res. 33: 2384-2394.

Nameki, N., Chattopadhyay, P., Himeno, H., Muto, A., and Kawai, G. 1999. An NMR and mutational analysis of an RNA pseudoknot of
Escherichia coli tmRNA involved in trans-translation. Nucleic Acids Res. 27: 3667-3675.

Nameki, N., Someya, T., Okano, S., Suemasa, R., Kimoto, M., Hanawa-Suetsugu, K., Terada, T., Shirouzu, M., Hirao, I., Takaku, H., et al. 2005. Interaction analysis between tmRNA and SmpB from Thermus thermophilus. J. Biochem. 138: 729-739.

Nissen, P., Kjeldgaard, M., Thirup, S., Polekhina, G., Reshetnikova, L., Clark, B.F., and Nyborg, J. 1995. Crystal structure of the ternary complex of Phe-tRNAPhe, EF-Tu, and a GTP analog. Science 270: 1464-1472.

Pokrovskaya, I.D. and Gurevich, V.V. 1994. In vitro transcription: Preparative RNA yields in analytical scale reactions. Anal. Biochem. 220: $420-423$.

Rudinger-Thirion, J., Giege, R., and Felden, B. 1999. Aminoacylated tmRNA from Escherichia coli interacts with prokaryotic elongation factor Tu. RNA 5: 989-992.

Shimizu, Y. and Ueda, T. 2002. The role of SmpB protein in transtranslation. FEBS Lett. 514: 74-77.

Shpanchenko, O.V., Zvereva, M.I., Ivanov, P.V., Bugaeva, E.Y., Rozov, A.S., Bogdanov, A.A., Kalkum, M., Isaksson, L.A., Nierhaus, K.H., and Dontsova, O.A. 2005. Stepping transfer messenger RNA through the ribosome. J. Biol. Chem. 280: 18368-18374.

Sundermeier, T.R., Dulebohn, D.P., Cho, H.J., and Karzai, A.W. 2005. A previously uncharacterized role for small protein $\mathrm{B}(\mathrm{SmpB})$ in transfer messenger RNA-mediated trans-translation. Proc. Natl. Acad. Sci. 102: 2316-2321.

Ushida, C., Himeno, H., Watanabe, T., and Muto, A. 1994. tRNA-like structures in 10Sa RNAs of Mycoplasma capricolum and Bacillus subtilis. Nucleic Acids Res. 22: 3392-3396.

Valle, M., Gillet, R., Kaur, S., Henne, A., Ramakrishnan, V., and Frank, J. 2003. Visualizing tmRNA entry into a stalled ribosome. Science 300: 127-130.

Williams, K.P. and Bartel, D.P. 1996. Phylogenetic analysis of tmRNA secondary structure. RNA 2: 1306-1310.

Wower, J. and Zwieb, C. 1999. The tmRNA database (tmRDB). Nucleic Acids Res. 27: 167.

Wower, J., Zwieb, C.W., Hoffman, D.W., and Wower, I.K. 2002. SmpB: A protein that binds to double-stranded segments in tmRNA and tRNA. Biochemistry 41: 8826-8836.

Wower, I.K., Zwieb, C., and Wower, J. 2004. Contributions of pseudoknots and protein $\mathrm{SmpB}$ to the structure and function of tmRNA in trans-translation. J. Biol. Chem. 279: 54202-54209.

Wower, I.K., Zwieb, C., and Wower, J. 2005. Transfer-messenger RNA unfolds as it transits the ribosome. RNA 11: 668-673.

Zwieb, C., Wower, I., and Wower, J. 1999. Comparative sequence analysis of tmRNA. Nucleic Acids Res. 27: 2063-2071. 

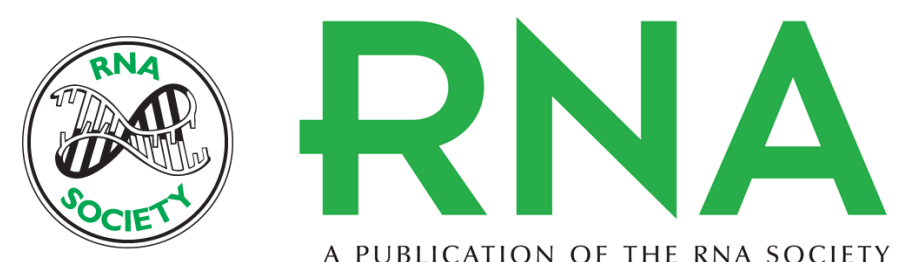

A PUBLICATION OF THE RNA SOCIETY

\section{Structure probing of tmRNA in distinct stages of trans-translation}

Natalia Ivanova, Magnus Lindell, Michael Pavlov, et al.

RNA 2007 13: 713-722 originally published online March 30, 2007

Access the most recent version at doi:10.1261/rna.451507

\section{References This article cites 45 articles, 17 of which can be accessed free at: http://rnajournal.cshlp.org/content/13/5/713.full.html\#ref-list-1}

License

Email Alerting Receive free email alerts when new articles cite this article - sign up in the box at the Service top right corner of the article or click here.

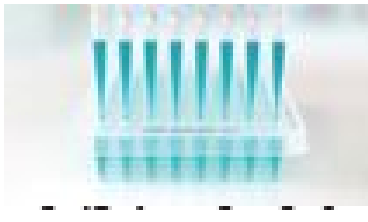

